\title{
1 Generation of isolated ultra-short attosecond pulses by coherent 2 control of the population of excited states
}

\author{
Hossein Z. Jooya ${ }^{1}$, Peng-Cheng $\mathrm{Li}^{2,3}$,Sheng-Lun Liao ${ }^{2}$, and Shih-I Chu ${ }^{1,2}$ \\ ${ }^{1}$ Department of Chemistry, University of Kansas, Lawrence, Kansas 66045, USA \\ ${ }^{2}$ Center for Quantum Science and Engineering, Department of Physics, National Taiwan \\ University, Taipei 10617, Taiwan \\ ${ }^{3}$ College of Physics and Electronic Engineering, Northwest Normal University, Lanzhou, Gansu \\ 730070, China
}

\begin{abstract}
We present an ab-initio theoretical investigation of the enhancement of ultra-broad super-continuum harmonic spectra by coherently controlling the electron quantum paths of the helium atom and $\mathrm{He}^{+}$ion. The time-dependent Schrödinger equation is solved by means of the time-dependent generalized pseudospectral method, allowing non-uniform and optimal spatial grid discretization and accurate and efficient propagation of the wave function in space and time. The population of the first two low-lying excited states of $\mathrm{He}^{+}$is selectively controlled by adding low intensity, high frequency laser pulses to a two color mid-infrared laser field. Although the intensity of the added field is weak, its high frequency makes the subsequent ionization probability from excited states become much larger than for the case of many photons (mid-IR filed), even though the intensity is smaller. We found that the intensity of the attosecond pulse generated by superposing a range of synchronized high harmonics is significantly enhanced by 20 orders of magnitude. Similar calculations have been performed for the neutral He atoms, We found that an intense and ultra-short isolated 18 as can be generated directly. To understand the underlying mechanism of such dramatic enhancement and the role of electron quantum paths, we perform wavelet time-frequency transform of high harmonic spectra. The results show that we can selectively control the domination of the two distinct long and short electron trajectories by controlling the population of different excited states.
\end{abstract}

Keywords: High-harmonic generation, Attosecond pulse, Coherent control 
The generation of ultra-short attosecond pulses and its application to the quantum control of electron dynamics in atomic, molecular, and condensed matter systems is a subject of much current interest in ultrafast science and technology in the last decade [1-10]. One of the most attractive candidates for the production of isolated attosecond pulses is by means of superposing a range of consecutive high harmonics in the super-continuum regime which can be synchronized and locked in phase, resulting in the production of coherent attosecond pulses [9-14]. Each harmonic is associated to both short and long electron trajectories that have the same return energy but very different return times [12]. When only single quantum path contributes to the $\mathrm{HHG}$, the single attosecond pulse can be obtained directly by superposing several harmonics which have almost the same emission time. Therefore the coherent control of these electron quantum paths is an efficient method for the production of the broadband supercontinuum harmonics spectra and isolated attosecond pulse [15-18]. As the two quantum trajectories have different ionizing and returning times, their harmonic phases often behave differently. As a result, the synthesis of several harmonics often leads to an irregular attosecond pulse. In order to obtain a single and coherent attosecond pulse, it is advantageous to select only one type of the two trajectories by coherent control of the electron quantum paths. Experimentally, the dominating trajectory is selected through phase matching by adjusting the position of the laser focus relative to the nonlinear medium [19]. Normally, only the HHG spectra from the short trajectories are observed on axis in the gas target experimentally [20]. In other words, the harmonics spectra from long trajectories are usually not dominant on axis after considering macroscopic propagation [21].

It has been shown that excited states play a role in high harmonic generation and enhancement [22-25]. In this work, we explore the feasibility of selective control over the domination of the two quantum pathways by populating different excited states of single $\mathrm{He}^{+}$ion to significantly enhance the HHG yield and cutoff extension, leading to the generation of intense and isolated ultra-short attosecond pulses. Although the present study focuses on the $\mathrm{He}^{+}$and $\mathrm{He}$ systems, similar idea can be extended to other more complex systems as well. For the neutral He atoms, we adopt the angular-momentum-dependent model potential recently developed [26] which allows for accurate description of the electronic structure of the ground and high-lying singly-excited states of He atoms.

We consider the interaction of an intense laser field with helium atom and $\mathrm{He}^{+}$ion by solving following the time-dependent Schrödinger equation (TDSE), (atomic units (a.u.) are used throughout this letter unless stated otherwise),

$i \frac{\partial}{\partial t} \psi(\boldsymbol{r}, t)=\widehat{H} \psi(\boldsymbol{r}, t)=\left[\widehat{H}_{0}+\widehat{V}(\boldsymbol{r}, t)\right] \psi(\boldsymbol{r}, t)$,

where $\widehat{H}_{0}$ is the unperturbed Hamiltonian for $\mathrm{He}^{+}$and $\widehat{V}(r, t)$ is the time-dependent atom-field interaction, with the laser field represented by $E(t)$,

$\widehat{V}(\boldsymbol{r}, t)=-\boldsymbol{E}(t) \cdot \boldsymbol{r}=-z E(t)$.

The time-dependent generalized pseudo-spectral (TDGPS) method [27] is used to solve the TDSE accurately and efficiently. The strength of this method is two-folded. First, it takes the advantage of generalized pseudo-spectral (GPS) technique for non-uniform optimal spatial discretization of the coordinates and the Hamiltonian using only a modest number of grid points. Second, it eliminates the undesirable fast-oscillating high-energy components by using a second-order split-operator technique in the energy representation. The time propagation of the wave function under this method is given as: 
$\psi(\boldsymbol{r}, t+\Delta t)$

$\cong \exp \left(-i \widehat{H}_{0} \frac{\Delta t}{2}\right) \times \exp \left[-i V\left(\boldsymbol{r}, \theta, t+\frac{\Delta t}{2}\right) \Delta t\right]$

$\times \exp \left(-i \widehat{H}_{0} \frac{\Delta t}{2}\right)+O\left(\Delta t^{3}\right)$.

The expectation value of the induced dipole, in acceleration form, is obtained from the timedependent wave function:

$d_{A}(t)=\left\langle\psi(\boldsymbol{r}, t)\left|\frac{d^{2} z}{d t^{2}}\right| \psi(\boldsymbol{r}, t)\right\rangle=\left\langle\psi(\boldsymbol{r}, t)\left|-\frac{z}{r^{3}}+E(t)\right| \psi(\boldsymbol{r}, t)\right\rangle$.

It is known that multi-color field schemes can remarkably enhance the cutoff energy of the HHG spectra and obtain a much shorter isolated attosecond pulse [28-29]. The core two-color driving laser fields used in the current study has the form as follows:

$E_{0}(t)=A_{1} f_{1}(t) \operatorname{Sin}\left(\omega_{1} t+\phi_{1}\right)+A_{2} f_{2}\left(t-\tau_{1}\right) \operatorname{Sin}\left[\omega_{2}\left(t-\tau_{1}\right)+\phi_{2}\right]$.

Here $A_{1}$ and $A_{2}$ are laser amplitudes, $f_{1}(t)$ and $f_{2}(t)$ are the Gaussian pulses with $F W H M_{1}=8 f s$ and $F W H M_{2}=5 \mathrm{fs}$ with peak intensities of $I_{1}=4.0 \times 10^{14} \mathrm{~W} / \mathrm{cm}^{2}$ and $I_{2}=1.6 \times 10^{14} \mathrm{~W} / \mathrm{cm}^{2}$, respectively. $\omega_{1}=0.0207$ a.u. $\left(\lambda_{1}=2200 \mathrm{~nm}\right)$ and $\omega_{2}=0.0434$ a.u. $\left(\lambda_{2}=1050 \mathrm{~nm}\right)$ are the laser frequencies. $\phi_{1}=\phi_{2}=0.0$ are the carrier-envelope phases (CEPs), and $\tau_{1}=0.55 T_{2}$ is the time delay between the two pulses, with $T_{2}=\frac{2 \pi}{\omega_{2}}$. This frequency ratio and the chosen time delay provide the optimized two-color mid-infrared laser pulse shape to significantly extend the HHG cutoff [26]. Jooya et. al. recently utilized Bohmian mechanics to demonstrate the effect of laser pulse shape on the characteristic properties of high-order harmonic generation and the sub-cycle ionization dynamics [3031].

In the present work, this laser field is further modified by adding a very weak high-frequency field as

$E(t)=E_{0}(t)+A_{3} f_{3}\left(t-\tau_{2}\right) \operatorname{Sin}\left[\omega_{3}\left(t-\tau_{2}\right)\right]$.

Here the peak intensity is $I_{3}=1.5 \times 10^{12} \mathrm{~W} / \mathrm{cm}^{2}$, and $F W H M_{3}=8 \mathrm{fs}$. These values are kept fixed in both of the upcoming calculations. When the population of the first excited state was targeted $\omega_{3}$ is set equal to the resonance frequency of the first excited state of $\mathrm{He}^{+}\left(\omega_{3}=1.51 \mathrm{a} \cdot u \cdot=\right.$ $30.15 \mathrm{~nm})$ with $\tau_{2}=-2.0 T_{1} \quad\left(T_{1}=\frac{2 \pi}{\omega_{1}}\right)$. Similarly for the second excited state of $\mathrm{He}^{+}$we use $\omega_{3}=1.78$ a.u. $=25.60 \mathrm{~nm}$, with $\tau_{2}=-1.1 T_{1}$. These are the energies required to populate the first and second excited states of $\mathrm{He}^{+}[32]$.

The introduced three-color laser fields are presented in figure 1(a). The corresponding HHG power spectra from $\mathrm{He}^{+}$exposed to each of these lasers is obtained, in the single-atom level, by the Fourier transformation of time-dependent dipole acceleration as follows: 
$P_{A}(\omega)=\left|\frac{1}{t_{f}-t_{i}} \frac{1}{\omega^{2}} \int_{t_{i}}^{t_{f}} d_{A}(t) e^{-i \omega t} d t\right|^{2}$

where $\omega$ is the harmonic frequency. The harmonic power spectra are presented in logarithmic scale as $S(\omega)=\log _{10} P_{A}(\omega)$. As can be seen in figure1(b), for the case of the optimized three-color laser field with $30 \mathrm{~nm}$ as the third color (dotted blue), the first cut-off harmonic order obtained from accurate TDSE calculations is occurred around $1200^{\text {th }}$ harmonic, and the cutoff of the second plateau is located at around $1500^{\text {th }}$ harmonic. According to the three-step model of HHG [33,34], the cutoff harmonic number in HHG is calculated by $N_{\text {cut-off }}=\left(I_{p}+3.17 U_{p}\right) / \omega_{1} \cong 1206$ (The ionization potential for $\mathrm{He}^{+}$is $I_{p}=2.0 \mathrm{a} . u$., and the ponderomotive potential of multicolor laser is obtained as $U_{p} \cong 7.25$ a.u.), in good agreement with the first HHG cutoff observed, while the cutoff of the second plateau is beyond $3.17 U_{p}$ (around $4 U_{p}$ ). The main feature of this result is the dramatic enhancement (by 15 orders of magnitude) in the HHG due to adding the high frequency component to the mid-IR two color laser. This high efficiency in the HHG production can be attributed to the much larger subsequent ionization from the excited state [35]. Extra calculations have also been performed in this research to confirm that the third color is essential in producing the high intensity and ultra-short isolated attosecond pulses. Applying a 30nm Gaussian shaped single color laser field provided clear evidence of the role of this term on dramatic increase of HHG. Also, using the presented two-color laser field on a pre-excited $\mathrm{He}^{+}$showed that although the intensity would be augmented, but the quality of the final attosecond pulse would be dramatically compromised by removing the third term from the driving laser field. Therefore, as proposed in this paper, the most efficient laser pulse to obtain the shortest and highest intensity attosecond outcome would be using the three-color laser pulse, with the controlled parameters presented above.
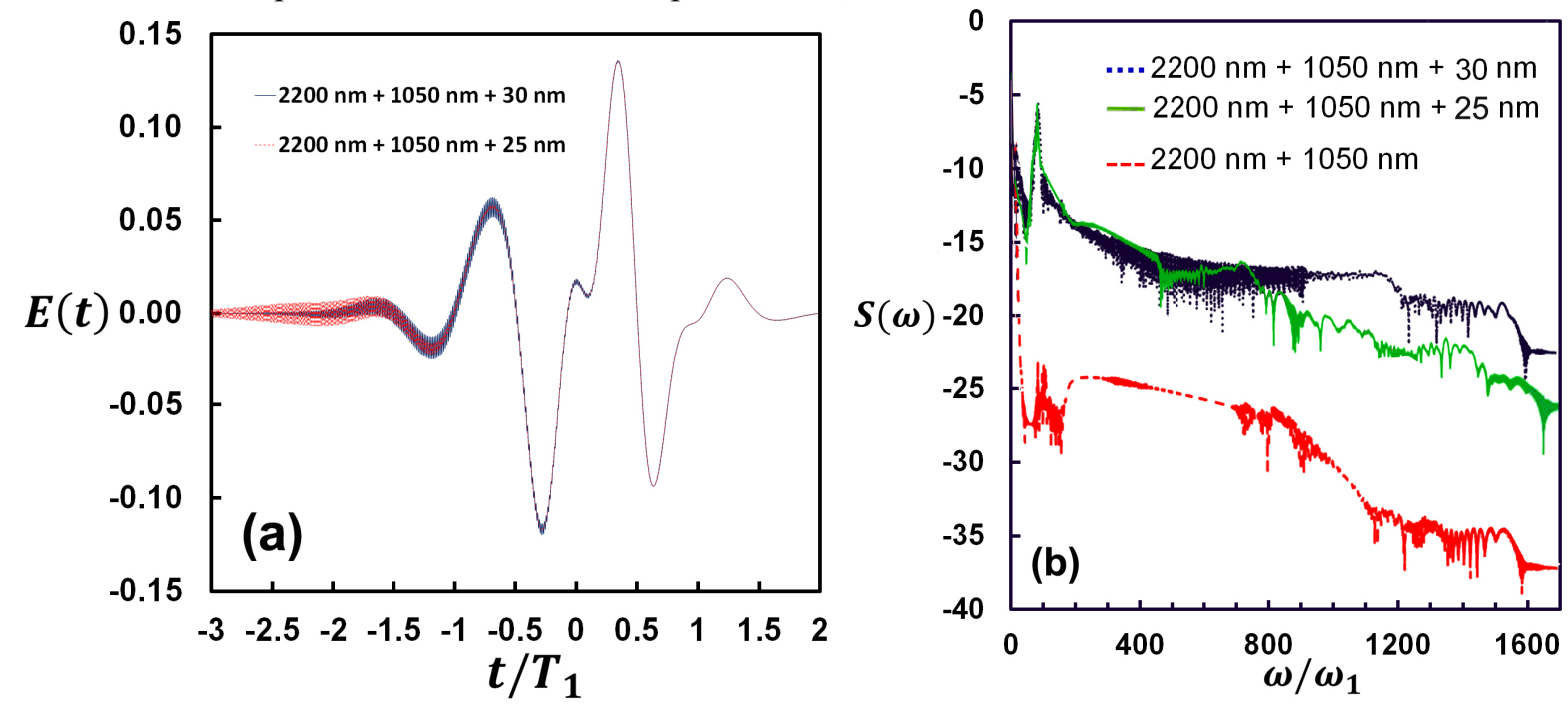

Figure 1. (a) The three-color, $E(t)$, driving laser fields, with $30 \mathrm{~nm}$ (solid blue) and $25 \mathrm{~nm}$ (dashed red) as the third color. The $x$-axis is time in optical cycle. (b) Comparison of the acceleration-form HHG power spectra generated of $\mathrm{He}^{+}$by applying the two color laser field (dashed red) and the three color laser fields, with $30 \mathrm{~nm}$ (dotted blue) and $25 \mathrm{~nm}$ (solid green) as the third color. For the case of the optimized threecolor laser field with $30 \mathrm{~nm}$ as the third color (dotted blue)The first cut-off appears around $1200^{\text {th }}$ harmonic order and the second one after $1500^{\text {th }}$ order. The $x$-axis is the harmonic order. The results are in 
The results for length gauge dipole moments, transition probabilities and ionization probabilities due to applying the proposed three-color fields on $\mathrm{He}^{+}$are presented in figure 2(a-c) respectively. In both threecolor laser cases pre-excitation due to single $\omega_{3}$ photon is followed by subsequent IR field ionization process. Figure 2(a) shows the time-dependent induced dipole moments of $\mathrm{He}^{+}$in length form. The strong electric response of $\mathrm{He}^{+}$to the three-color lasers, comparing to its magnitude when two-color laser is applied, is clearly demonstrated in this figure. Generally, the dipoles have the same sign as the field does. Thus for the second excited state (dashed red) the negative and positive peaks in the dipole moment correspond to the peaks of the field around -1.1 and -0.7 optical cycle (o.c.), see figure 1(a). Another observation is that the large dipoles are caused by the population in the excited states which have larger polarizability. Consequently, the peaks of the dipole moment exist when there is substantial population in the second excited state. This is in agreement with the transition probability to the excited states (in this case first or second excited states), $\left\langle\varphi_{\text {Excited state }} \mid \psi(t)\right\rangle$, presented in figure 2(b). At larger times, the population in the second excited state is decreased because of ionization, see figure 2(c). That is why we do not see other peaks in the dipole moment at larger times. For the first excited state (solid blue), the population is trapped for longer time. According to figure 2(c), ionization occurs at later times; also there is some burst in the population just before the pulse peak. That is why we can still see the negative peak in the dipole moment corresponding to the main negative peak of the field (at -0.3 o.c. ). 

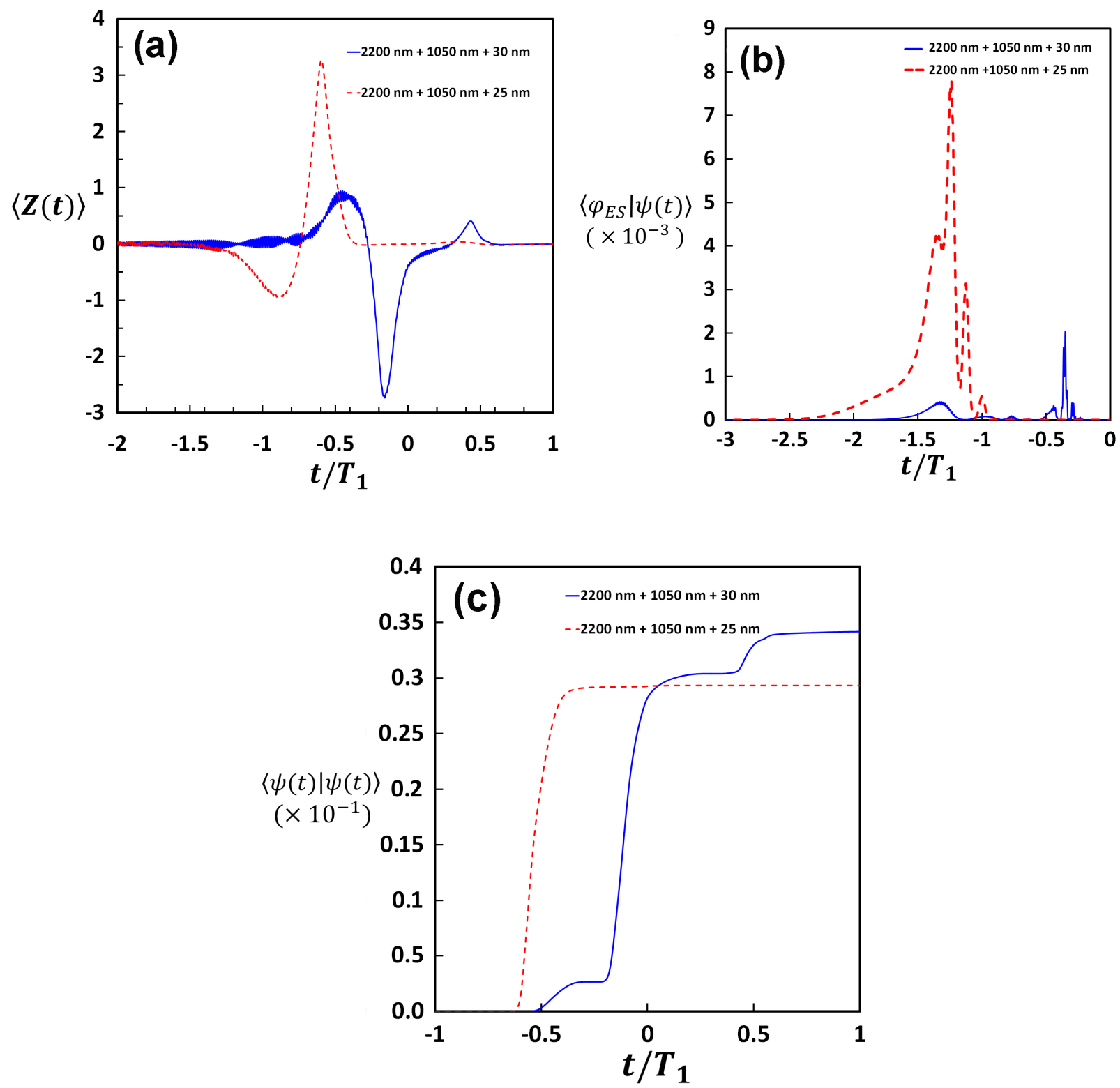

171

172 Figure 2. (a) Time-dependent induced dipole moments of $\mathrm{He}^{+}$in length form, in atomic units (a.u.). (b)

173 Transition probability amplitude to first (solid blue) and second (dashed red) excited states, $\varphi_{E s}$, of $\mathrm{He}^{+}$ under the three-color laser fields, and (c) Corresponding ionization probabilities of $\mathrm{He}^{+}$under the optimized three-color laser fields.

178 the wavelet spectrum as the following [36],

179

$A(t, \omega)=\int \sqrt{\omega} d_{A}\left(t^{\prime}\right) W\left[\omega\left(t^{\prime}-t\right)\right] d t^{\prime}=d_{\omega}(t)$ 
Here $W\left[\omega\left(t^{\prime}-t\right)\right]$ is the mother wavelet. For the harmonic emission, a natural choice of mother wavelet is given by the Morlet wavelet [36],

$W(x)=\frac{1}{\sqrt{\tau}} \exp (i x) \exp \left(-x^{2} / 2 \tau^{2}\right)$

In figure 3, the wavelet time-frequency profiles of the HHG spectra of $\mathrm{He}^{+}$driven by the two and threecolor laser fields are presented. Emission of high-order harmonics can be qualitatively explained by the three-step model $[33,34]$. According to this model, the recombination times of the different electron trajectories determine the emission times of the different XUV frequencies and their possible synchronization. The returning electron will emit harmonic photons by radiative recombination with the parent ionic core. The highest energy electrons are made when the electron is accelerated by a maximal of the field amplitude and thus photons with the highest energy are produced [20,37]. When only a single quantum path contributes to the HHG, the single attosecond pulse can be obtained directly by superposing several harmonics which have almost the same emission time. One can clearly see in figure 3(a) that, by applying the two-color laser field, the $600^{\text {th }}$ to $1200^{\text {th }}$ harmonics would be constructed by contributions from both long and short-trajectory electrons (the red zones correspond to higher electron population contributing to the corresponding harmonics. In this range of harmonics the population is distributed between both short and long trajectory electrons). The consequence is that, as we will show later, this harmonics range cannot serve as a good candidate to obtain an isolated attosecond pulse from. On the other hand, when the first excited state is populated by applying the three-color, figure 3(b), the domination of two distinct mechanisms for electron trajectories at different times is achieved. The two distinct peaks in the wavelet diagram correspond to the two separated plateaus observed in figure 1(b). The peak between -0.5 to 0.0 optical cycle in the wavelet diagram indicates that during this radiation time, the short-trajectory electrons emit in a shorter time range and contribute to each harmonic order near the first cutoff region. However, the later peak which occurs between 0.0 to 0.5 optical cycles represents the emission of the long-trajectory electrons. These electrons emit over a relatively longer time range and contribute to the harmonics near the second cutoff region, from $1200^{\text {th }}$ to $1480^{\text {th }}$. The magnificent difference between the intensity of these two peaks can be better understood by looking at intensities of the corresponding electric responses shown in figure 2(a). Another novel feature of the here presented results can be observed by comparing the wavelets due to populating the first excited state, figure 3(b), with the case that the second excited state is being populated, figure 3(c). One can clearly observe the domination of two distinct mechanisms for electron trajectories between -0.5 to 0.0 optical cycle under different laser fields. When the first excited state is being populated, figure 3(b), the dominant trajectory is characterized by an electron returning close to -0.5 optical cycle (short trajectory). The two distinct peaks in this wavelet diagram correspond to the two separated plateaus observed in figure 1(b) (solid blue plot). On the other hand, as illustrated by the wavelet in figure 3(c), when the population of the second excited state is under control, the long trajectory electrons are responsible and dominant at the same time range (between -0.5 to 0.0 optical cycle). The three separated plateaus observed in figure 1(b) (dashed red plot) correspond to the three distinct peaks in this wavelet. In both cases the returning electron will emit harmonic photons by radiative recombination with the parent ionic core. The highest energy electrons are made when the electron is accelerated by a maximal of the field amplitude and thus photons with the highest energy are produced [20,38]. 

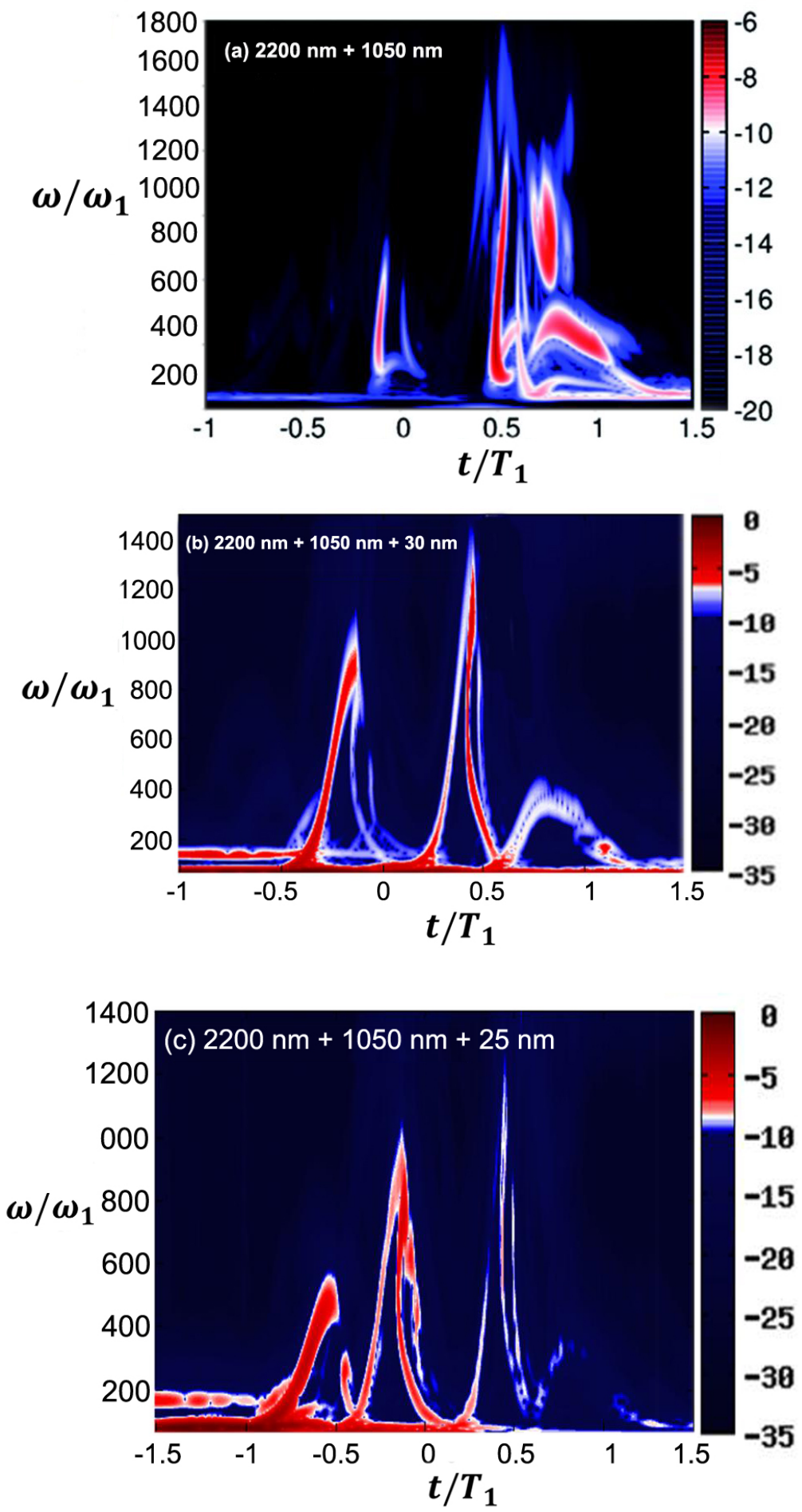

Figure 3. Wavelet time-frequency profile of the HHG power spectra of $\mathrm{He}^{+}$driven by the (a) Two-color and (b,c) Optimized three-color laser field. The $x$-axis is time in optical cycle and the $y$-axis is the harmonic order. The results are in atomic units (a.u.). 
The emission of all the consecutive harmonics in the cutoff, provided that they have similar coherent 229 properties, can in principle be superimposed with each other to produce a stronger radiation emission. In 230 other words, synchronization of a given range of harmonics can take place provided that each individual 231 harmonic has similar dynamical phase (also called the spectral phase) during the emission process. The 232 attosecond pulse, $I(t)$, is calculated by integrating the supercontinuum in the HHG power spectrum,

$I(t)=\left|\sum_{q} a_{q} e^{i q \omega t}\right|^{2}$

234 Here $a_{q}=\int d_{A}(t) e^{-i q \omega t} d t[38]$.

235 Figure 4(a) and (b) present the isolated attosecond pulses obtained from the interaction of the two-color 236 and the first excited state populating three-color laser fields with $\mathrm{He}^{+}$, respectively. The weak 40as pulse 237 presented in figure 4(a) is obtained by superposing harmonics with concentrated emission time near the 238 first cutoff, from the $1100^{\text {th }}$ to $1280^{\text {th }}$ order. Figure 4(b) presents a $24 a$ s pulse generation which is 239 obtained by superposing a range of harmonics near the second cutoff, from $1200^{\text {th }}$ to $1480^{\text {th }}$ order. The 240 intensity and pulse duration of this pulse are significantly improved by introducing the third high241 frequency term to the laser field. The other observation is that, although in both three-color laser cases the 242 width of the produced pulses are the same, 24as, but the yield of the pulse produced by the laser field that 243 controls the population of the first excited state is 20 times higher than the case that the second excited 244 state is under control. This enhancement in HHG and, in turn, in the yield of the generated attosecond 245 pulse is due to the delayed stepwise ionization of the electrons trapped in the first excited states for a 246 longer time. 

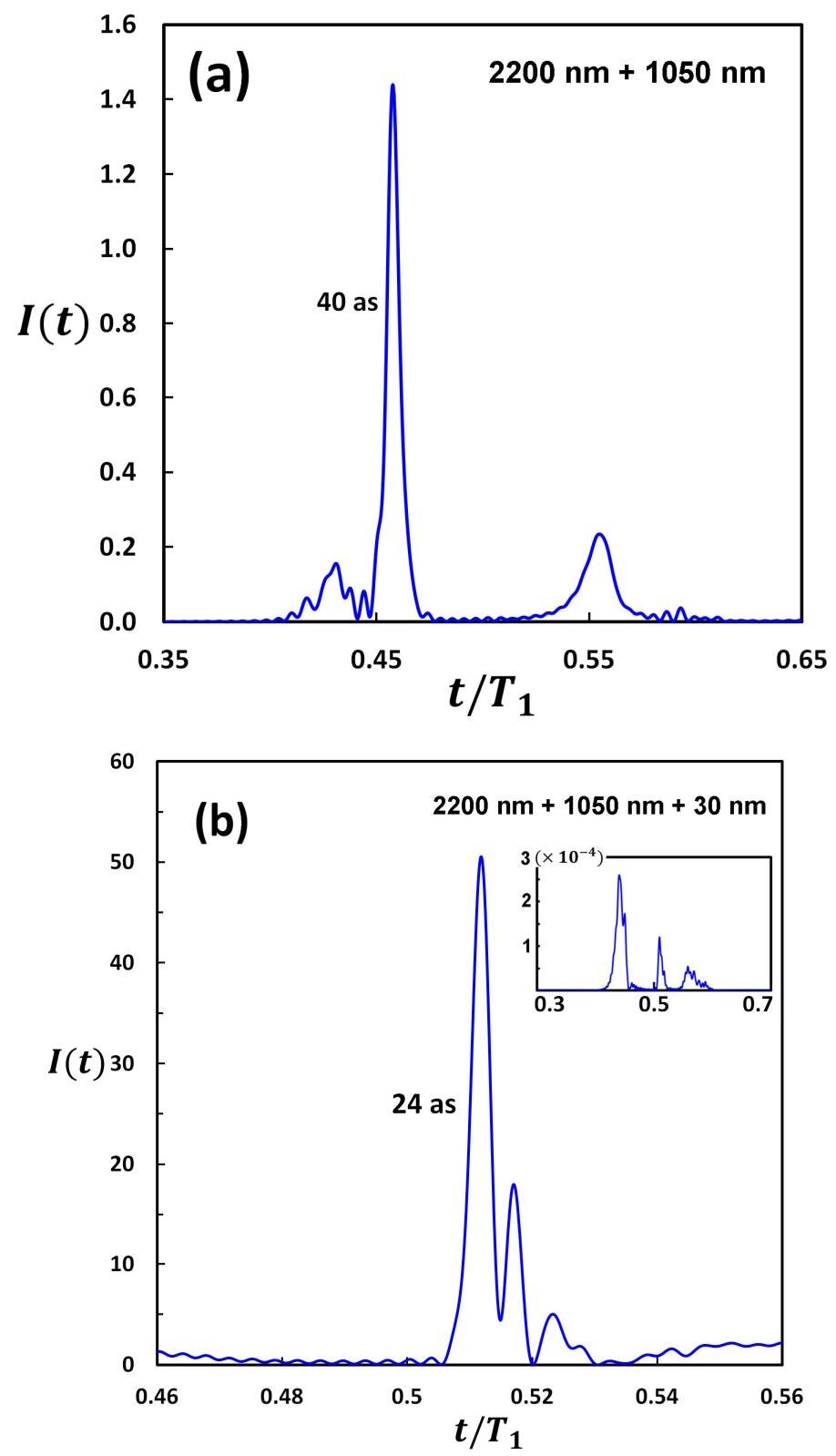

Figure 4. Isolated attosecond pulses produced by superposing the harmonics near the HHG cutoffs. (a) A 40 as pulse obtained from the superposition of harmonics from $1100^{\text {th }}$ to $1280^{\text {th }}$ harmonic order when $\mathrm{He}^{+}$ is exposed by two-color driving laser field. (b) An intense 24as pulse obtained from the superposition of $1200^{\text {th }}$ to $1480^{\text {th }}$ harmonics of $\mathrm{He}^{+}$under optimized three-color driving laser field. The inset is the same result from applying the two-color laser field. The results are in atomic units (a.u.).

Similar calculations have been performed for the neutral He atom. To obtain an accurate calculation of the harmonic spectra of $\mathrm{He}$, a recently developed angular-momentum-dependent model potential is utilized [26]. In figure 5(a), we present the HHG power spectrum from $\mathrm{He}$ atom driven by the optimizing three-color laser pulses. In particular, we added a 50nm uv pulse to the previously introduced two-color 
laser field; because its photon energies coincide with single-electron resonant energy from the ground state to the second excited state of He atom. Other laser parameters used are the same as in Figure 1. For reference, the HHG of He atom subject to the two-color laser field is also presented (red dashed line). It is clearly seen that the plateau in HHG is enhanced and a broadband super-continuum near cutoff is obtained from the He atom in the optimized three-color laser pulses. The reason is that the HHG emission of the long quantum trajectories is enhanced and the short one is suppressed by adding a uv laser pulse. As a result, an isolated 18 as pulse is produced directly for the optimizing three-color laser pulse case. Furthermore, the intensity of attosecond pulse is enhanced and the duration is shorter than that of the twocolor case. Therefore, the proposed method can be extended to other systems and can provide an efficient method for the production of isolated ultra-short attosecond pulses.
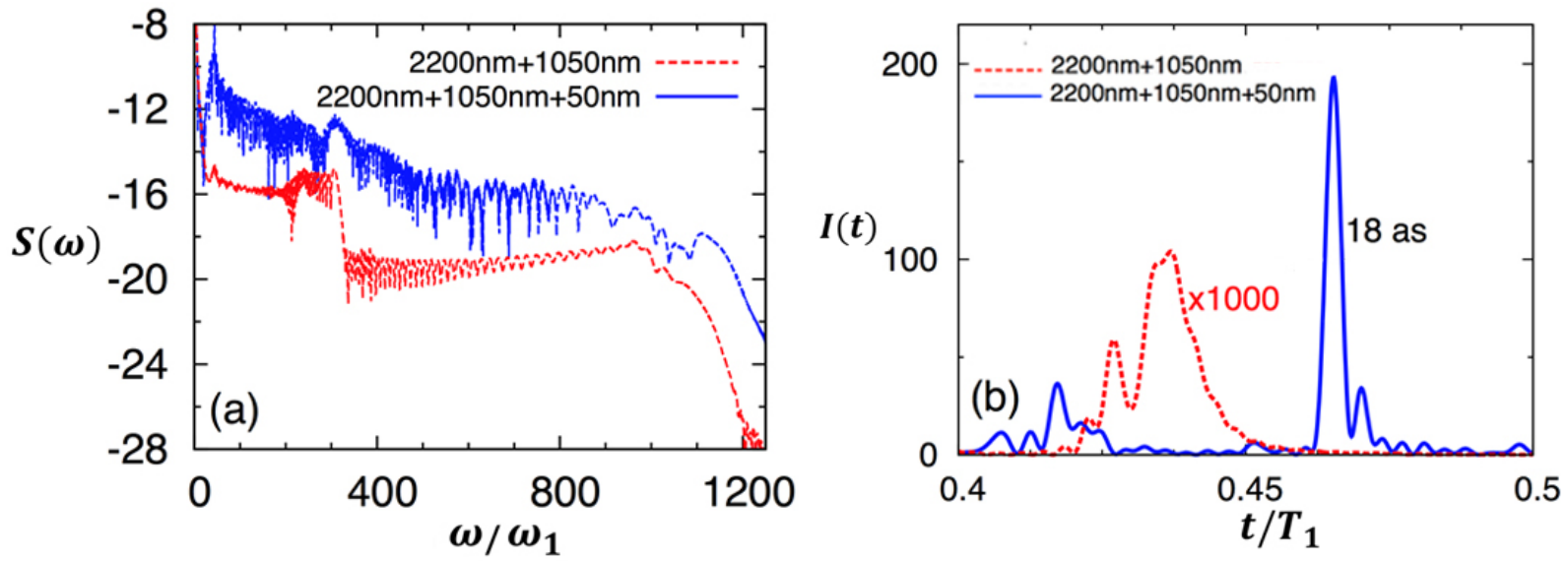

Figure 5. HHG power spectrum generated by applying two- and three-color lasers pulses on He atoms. The $\mathrm{x}$-axis is the harmonic order. (b) An intense 18as pulse generated by superposing the harmonics from 480th to 780th harmonics of He atom in the optimizing three-color laser pulse (blue line). For comparison, attosecond pulse generation in optimizing two-color laser fields is also presented (red dashed line). The $\mathrm{x}$ axis is the time in optical cycle.

In conclusion, we have presented in this article an efficient scheme to produce an isolated ultra-short attosecond pulse from helium atom and $\mathrm{He}+$ ion subject to optimized three-color laser fields. We found that the resonance excitation of excited states by adding an optimized very weak high-frequency component to a mid-IR laser, in turn, enhances the yield of HHG dramatically, leading to the generation of ultra-short and intense attosecond pulse. It is also shown that one can selectively control the domination of the two distinct long and short electron trajectories by controlling the population of different excited states. We believe that the proposed method is general and can be extended to other more complicated systems as well for the efficient generation of more intense and isolated ultra-short attosecond pulses for the future. Also, the extension of this work to investigate the effect of the macroscopic propagation (phase-matching) on the super-continuum harmonic spectra and the subsequent attosecond-pulse generation is in progress. In conclusion, we have presented in this article an efficient scheme to produce an isolated ultra-short attosecond pulse from helium atom and $\mathrm{He}^{+}$ion subject to optimized three-color laser fields. We found that the resonance excitation of excited states by adding an optimized very weak high-frequency component to a mid-IR laser, in turn, enhances the yield of HHG dramatically, leasing to the generation of ultra-short and intense attosecond pulse. It is also shown that one can selectively control the domination of the two distinct long and short electron trajectories by controlling the population of different excited states. We believe that the proposed method is general and can be extended to other more complicated systems as well for the efficient generation of more intense 
and isolated ultra-short attosecond pulses for the future. Also, the extension of this work to investigate the effect of the macroscopic propagation (phase-matching) on the super-continuum harmonic spectra and the subsequent attosecond-pulse generation is in progress.

\section{Acknowledgment}

This work was partially supported by the Chemical Sciences, Geosciences and Biosciences Division of the Office of Basic Energy Sciences, Office of Sciences, US Department of Energy. We also acknowledge partial support from the Ministry of Science and Technology of Taiwan and National Taiwan University (Grant No. NTU-104R104021 and NTUERP-104R8700-2). P. C. L. acknowledges partial support from National Natural Science Foundation of China (Grants No. 11364039 and No. 11465016), Natural Science Foundation of Gansu Province (Grant No. 1308RJZA195), and Education Department of Gansu Province (Grant No. 2014A-010). The authors would like to thank D. A. Telnov for valuable communications.

\section{References}

[1] M. Chini, K. Zhao, Z. Chang, Nature Photonics. 8 (2014) 178.

[2] M. Chini, X. Wang, Y. Cheng, Y. Wu, D. Zhao, D. A. Telnov, S. I. Chu, Z. Chang, Nature Sci. Rep. 3 (2013) 1105.

[3] K. T. Kim, C. Zhang, A. D. Shiner, S. E. Kirkwood, E. Frumker, G. Gariepy, A. Naumov, D. M. Villeneuve, P.B. Corkum, Nature Physics 9 (2013) 159.

[4] X. Wang, M. Chini, Q. Zhang, K. Zhao, Y. Wu, D. A. Telnov, S. I. Chu, Z. Chang, Phys. Rev. A 86 (2012) 021802(R).

[5] Z. H. Chang, P. B. Corkum, J. Opt. Soc. Am. B 27 (2010) 9.

[6] J. Itatani, J. Levesque, D. Zeidler, H. Niikura, H. Pepin, P. B. Corkum, D. M. Villeneuve, Nature 432 (2004) 867.

[7] R. Kienberger, M. Hentsche, M. Uiberacker, Ch. Speilmann, M. Kitzler, A. Scrinzi, M. Wieland, Th. Westerwalbesloh, U. Kleineberg, U. Heinzmann, M. Drescher, F. Krausz, Science 297 (2002) 1144.

[8] M. Drescher, M. Hentschel, R. Kienberger, M. Uiberacker, V. Yakovlev, A. Scrinzi, Th. Westerwalbesloh, U. Kleineberg, U. Heinzmann, F. Krausz, Nature 419 (2002) 803.

[9] P. Antoine, A. L'Huillier, M. Lewenstein, Phys. Rev. Lett. 77 (1996) 1234.

[10] S. F. Zhao, X. X. Zhou, P. C. Li , Z. Chen, Phys. Rev. A 78 (2008) 063404.

[11] M. B. Gaarde, K. J. Schafer, Phys. Rev. Lett. 89 (2002) 213901.

[12] Y. Mairesse, et. al., Phys. Rev. Lett. 93 (2004) 163901.

[13] K. Zhao, A. Zhang, M. Chini, Y. Wu, X. Wang, Z. Chang, Opt. Lett. 37 (2012) 3891.

[14] G. Sansone, L. Poletto, M. Nisoli, Nature Photonics 5 (2011) 655.

[15] G. Sansone, et al., Science 314 (2006) 443.

[16] K. J. Schafer, M. B. Gaarde, A. Heinrich, J. Biegert, U. Keller, Phys. Rev. Lett. 92 (2004) 023003.

[17] M. Fieb, B. Horvath, T. Wittmann, W. Helml, Y. Cheng, Z. Zeng, Z. Xu, A. Scrinzi, J. Gagnon, F. Krausz, R. Kienberger, New J. Phys. 13 (2011) 033031.

[18] H. Z. Jooya, D. A. Telnov, P. C. Li, S. I Chu, Phys. Rev. A 91 (2015) 063412.

[19] M. B. Gaarde, J. L.Tate, K. J. Schafer, J. Phys. B 41 (2008) 132001.

[20] Y. Mairesse, et al, Science 302 (2003) 1540.

[21] P. C. Li, S. I. Chu, Phys. Rev. A 86 (2012) 013411.

[22] P. M. Paul et. al, Phys. Rev. Lett. 94 (2005) 113906.

[23] Z. Zheng, R. Li, Y. Cheng, W. Yu, Z. Xu, Phys. Scr. 66 (2002) 321.

[24] J. G. Chen, Y. J. Yang, S. L. Zeng, H. Q. Liang, Phys. Rev. A 83 (2011) 023401.

[25] J. M. N. Djiokap, A. F. Starace, Phys. Rev. A 88 (2013) 053412. 
[26] P. C. Li, C. Laughlin, S. I. Chu, Phys. Rev. A 89 (2014) 023431.

347 [27] X. M. Tong, S. I. Chu, Chem. Phys. 217 (1997) 119.

348 [28] L. Feng, T. Chu, Phys. Lett. A 375 (2011) 3641.

349 [29] R. F. Lu, H. X. He, Y. H. Guo, K. L Han, J. Phys. B 42 (2009) 225601.

350 [30] H. Z. Jooya, D. A. Telnov, P. C. Li, S. I . Chu, J. Phys. B: At. Mol. Opt. Phys. 48 (2015) 195401.

351 [31] H. Z. Jooya, D. A. Telnov, P. C. Li, S. I . Chu, Phys. Rev. A 91 (2015) 063412.

352 [32] K. Ishikawa, Phys. Rev. Lett. 91 (2003) 043002.

353 [33] P. B. Corkum, Phys. Rev. Lett. 71 (1993) 1994.

354 [34] K. C. Kulander, K. J. Schafer, J. L. Krause, Proceedings of the Workshop on Super-Intense Laser 355 Atom Physics (SILAP) III, edited by Piraux P(Plenum Press, New York) 316 (1993) 95.

356 [35] M. Y. Emelin, M. Y. Ryabikin, A. M. Sergeev, M. D. Chernobrovtseva, T. Pfeifer, G. Gerber, 357 Europhys. Lett. 69 (2005) 913.

358 [36] X. M. Tong, S. I. Chu, Phys. Rev. A 61 (2000) 021802(R).

359 [37] J. J. Carrera, X. M. Tong, S. I. Chu, Phys. Rev. A 74 (2006) 023404.

360 [38] I. L. Liu, P. C. Li, S. I. Chu, Phys. Rev. A 84 (2011) 033414. 